\title{
Does Caffeine Intake Increase the Incidence of Tinnitus? A Systematic Review
}

\author{
Sattam M. Aljuaid ${ }^{10}$ Ahmad A. Mirza ${ }^{2(1)}$ Lura A. Habib ${ }^{3(0)}$ Lujain A. AlHarthi ${ }^{30}$ \\ Bashayer M. Alansari ${ }^{3}$ Bashaier G. AlQahtani ${ }^{30}$ Youssef A. Althobaiti ${ }^{4}$ \\ 1 Department of Otolaryngology, Head and Neck Surgery, King \\ Abdulaziz Specialist Hospital, Taif, Saudi Arabia \\ 2 Department of Otolaryngology, Head and Neck Surgery, Faculty of \\ Medicine in Rabigh, King Abdulaziz University, Jeddah, Saudi Arabia \\ Address for correspondence Ahmad A. Mirza, MBBS, MSc, \\ Department of Otolaryngology, Head and Neck Surgery, Faculty of \\ Medicine in Rabigh, King Abdulaziz University, P.O Box 80205, Jeddah \\ 21589, Saudi Arabia (e-mail: aamirza1@kau.edu.sa). \\ ${ }^{3}$ College of Medicine, Taif University, Taif, Saudi Arabia \\ ${ }^{4}$ Department of Internal Medicine - Neurology Section, King \\ Abdulaziz Specialist Hospital, Taif, Saudi Arabia \\ Int Arch Otorhinolaryngol 2021;25(4):e628-e632.
}

\begin{abstract}
Introduction Excessive caffeine intake has been thought to be a contributory factor for tinnitus. However, there has been no systematic review to elucidate the causal relationship between caffeine intake and the incidence of tinnitus.

Objectives We performed the current review aiming at evaluating the evidence from the current literature for the relationship between caffeine intake and the incidence of tinnitus.

Data Synthesis Databases including PubMed, Scopus, and Google scholar were searched for relevant articles. A total of 142 studies were screened for eligibility, of which four articles met our inclusion criteria: two were prospective cohorts and two were cross-sectional studies. Although one study found no association between caffeine consumption and the incidence of tinnitus, an inverse relationship was reported by two population-based studies. Concerning patients with preexisting

Keywords

- caffeine

- epidemiology

- tinnitus

- incidence

- severity

- review tinnitus, reduction of caffeine intake in a subset who consumed $150 \mathrm{ml}$ to $300 \mathrm{ml} /$ day of coffee yielded a favorable outcome in tinnitus severity. However, those with higher dose intake were less prone to have improvement in the severity of tinnitus.

Conclusion Although the current review was inconclusive, it appears that the incidence of tinnitus in previously unaffected individuals might be prevented by a high dose of caffeine intake. However, in preexisting tinnitus, a high dose of caffeine may adversely interfere with the efficacy of caffeine reduction.
\end{abstract}

\section{Introduction}

Tinnitus is an otologic manifestation characterized by the perception of noise or ringing in the ears. It affects 12 to $30 \%$ of the population, and the elderly are at highest risk for the disease. ${ }^{1,2}$ Although it is associated with disorders of the auditory and/or somatosensory systems, its precise etiology is poorly understood. The treatment of tinnitus is generally not promising, particularly in the absence of underlying cause. ${ }^{3,4}$ Peripheral nerve injuries, increased activity in the received

June 15,2020

accepted

September 1, 2020

published online

November 30, 2020
DOI https://doi.org/ 10.1055/s-0040-1718965. ISSN 1809-9777.

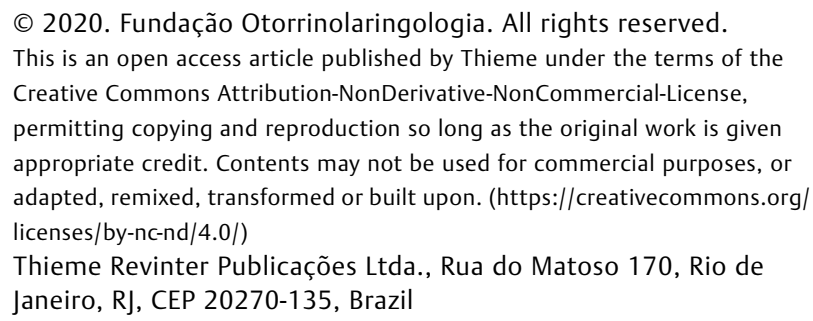


auditory pathways, central nervous system (CNS) lesions, and neurotransmitter abnormalities are implicated in the pathogenesis of the disease. ${ }^{5,6}$

Caffeine has a diverse effect on human organism, and an excessive amount of caffeine consumption might cause deleterious impact on health. ${ }^{7}$ It has been reported to directly enhance the release of glutamate and increase subsequent nerve activity. ${ }^{8}$ In addition to this glutamatereleasing effect, caffeine has an antagonistic influence on adenosine receptors, making it an effective treatment in some pain and neurodegenerative diseases. ${ }^{9}$

Given that caffeine has an excitatory effect on vestibular cells, ${ }^{10}$ many studies have examined the relationship between caffeine consumption and the incidence of tinnitus. Although some reports have shown a positive relationship, ${ }^{5,11}$ others failed to elucidate any association. ${ }^{12,13}$ Therefore, we systematically reviewed the current literature with an in-depth evaluation of the relationship between caffeine consumption and tinnitus.

\section{Review of Literature}

We used PubMed, Scopus, and Google Scholar electronic databases to carry out the literature search. The search terms "caffeine" AND "tinnitus" were used to identify original articles. Two authors searched these databases independently using the same search terms. After all original articles were identified, a further search was conducted to include relevant references from eligible studies. Using the Preferred Reporting Items for Systematic Reviews and Meta-Analyses
(PRISMA) flowchart, potentially relevant articles were identified after duplicates were excluded ( - Fig. 1). We included human studies in English without restrictions on gender, place, or time. Only studies that were performed among adult individuals who experienced the symptom of tinnitus were included. Each included study examined the relationship between caffeine intake and tinnitus incidence. We excluded reports limited to specific tinnitus diseases (e.g., Meniere disease). Case reports, cases series, and duplicate studies were also excluded. A standardized data extraction form was used. The primary outcome assessment for each study was the relationship of caffeine intake with the incidence of tinnitus. Other extracted data were authors' names, study year, study design, population characteristics, exposure definition, outcome measured, adjusted variables, measure of associations, and authors' conclusions.

-Fig. 1 illustrates the study selection process, and the number of articles identified in each step were shown in the same flowchart. A total of 139 articles were initially identified. A further search including references revealed three additional articles. After exclusion of 18 duplicates, 124 articles underwent title and abstract review. The qualitative analysis was performed on 4 studies: 2 prospective cohort studies $^{14,15}$ and 2 cross-sectional studies. ${ }^{12,16}$ The main characteristics of these studies are presented in - Table 1 . Of the four studies, one was from Brazil, two were from the United Kingdom, and one was from South Korea.

Three out of the four studies showed inverse relationships between tinnitus and caffeine consumption. ${ }^{14-16}$ A longitudinal experiment from the nurse health study II, in which

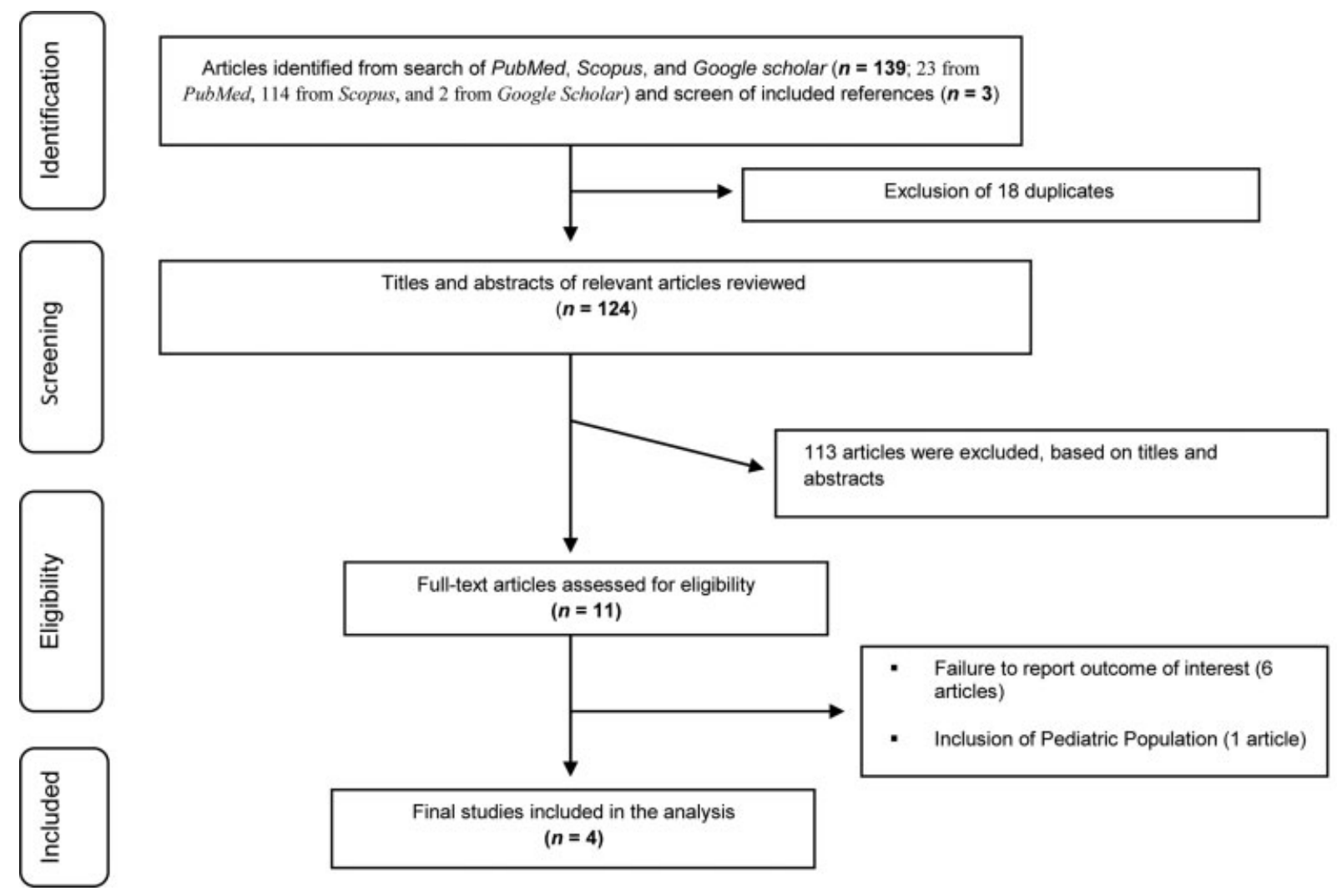

Fig. 1 Flowchart diagram of article selection. 


\begin{tabular}{|c|c|c|c|c|}
\hline 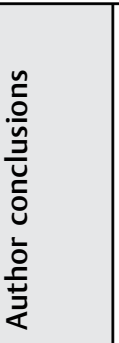 & 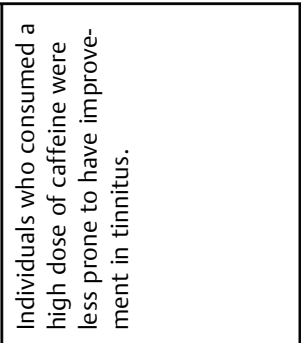 & 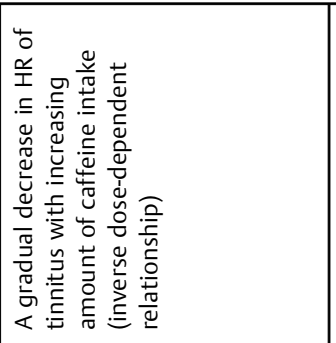 & 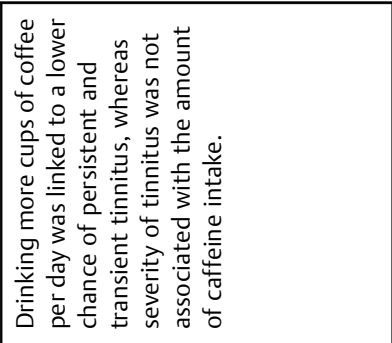 & 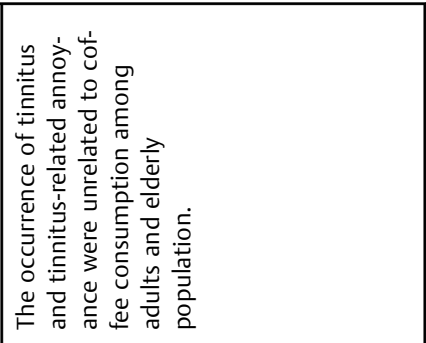 \\
\hline 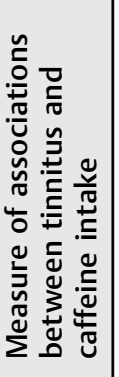 & 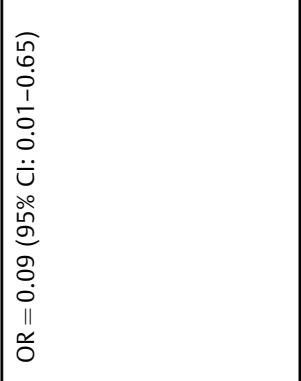 & 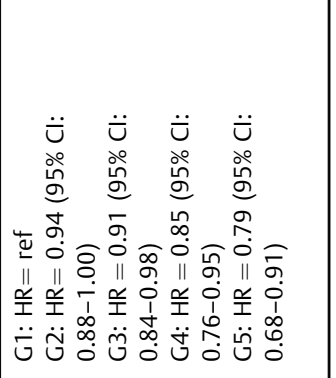 & 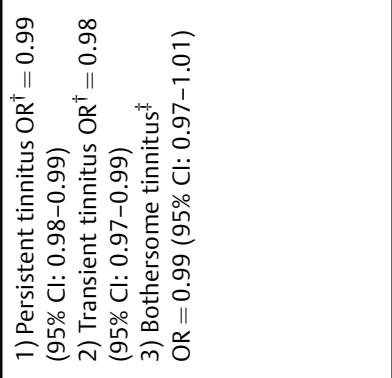 & 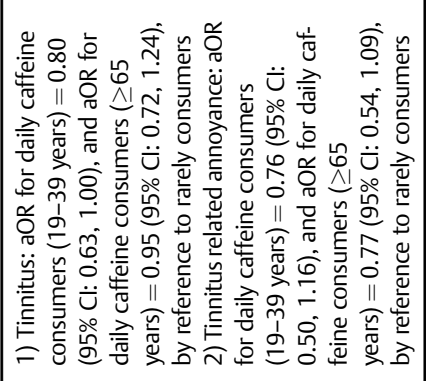 \\
\hline 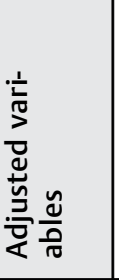 & $\begin{array}{l}0 \\
\text { Oे } \\
2\end{array}$ & 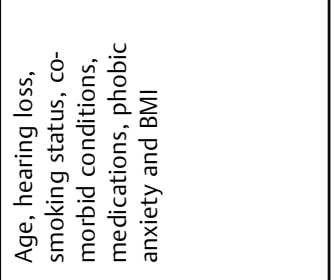 & 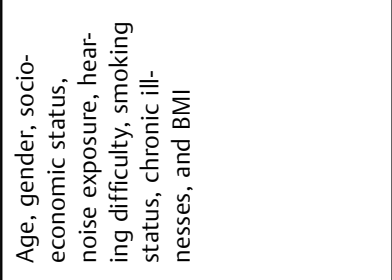 & 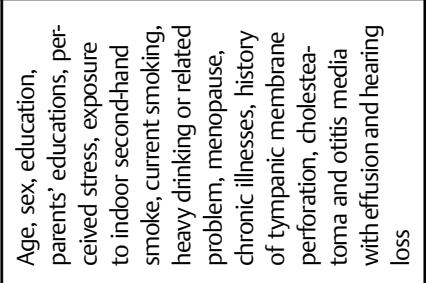 \\
\hline 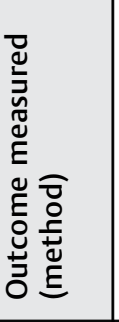 & 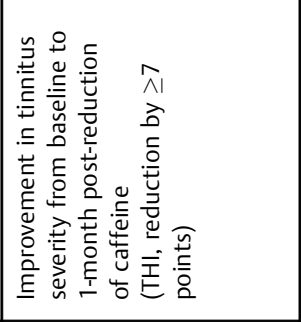 & 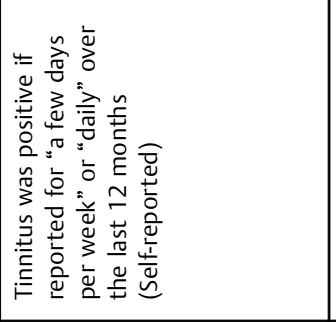 & 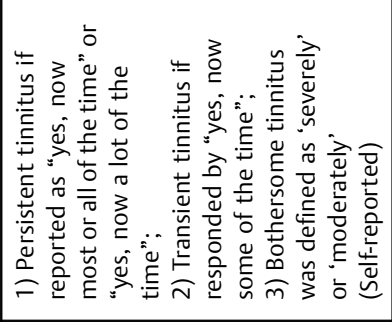 & 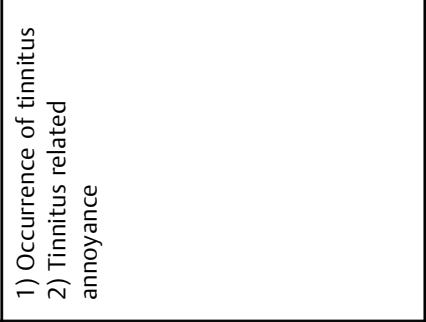 \\
\hline 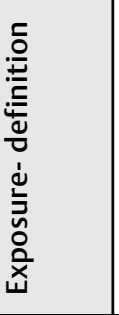 & 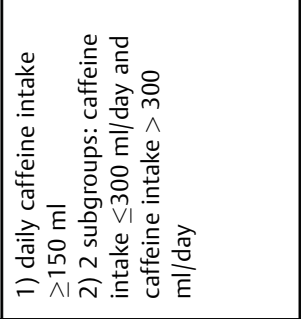 & 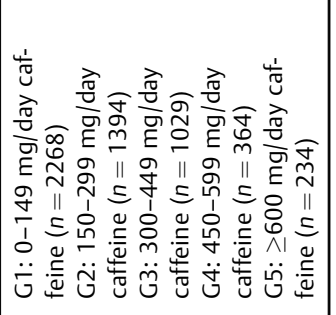 & 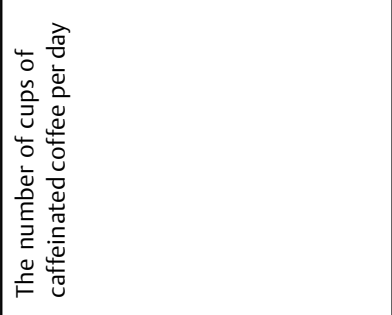 & 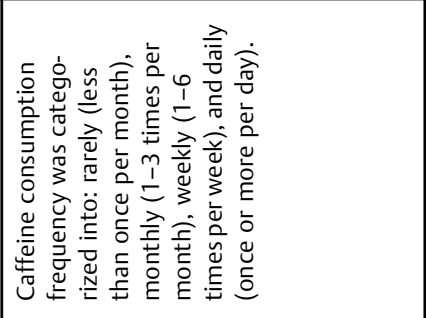 \\
\hline 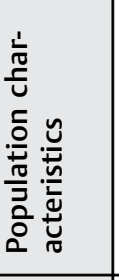 & 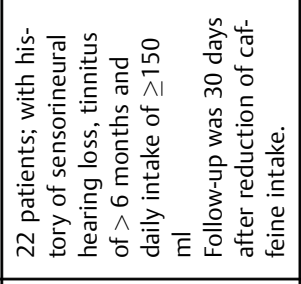 & 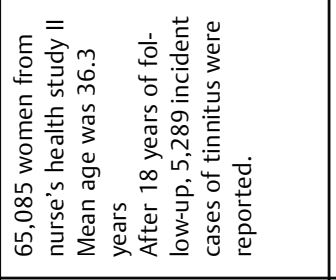 & 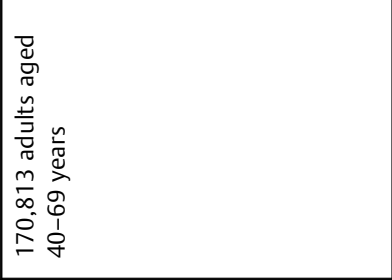 & 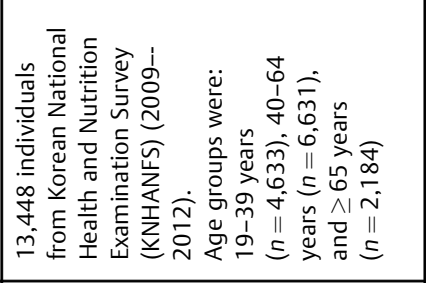 \\
\hline 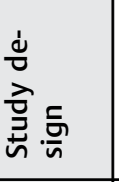 & 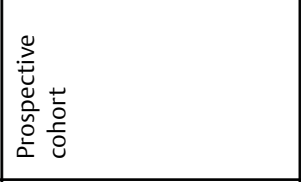 & 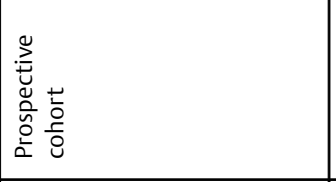 & 离 & 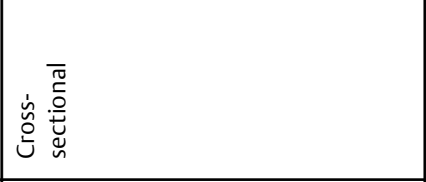 \\
\hline जे & 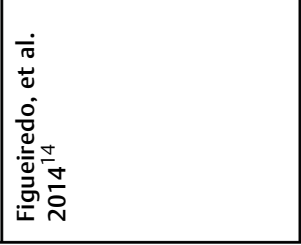 & 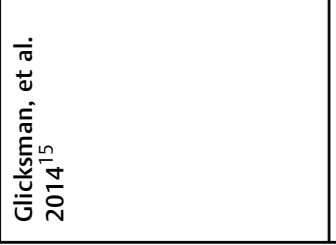 & 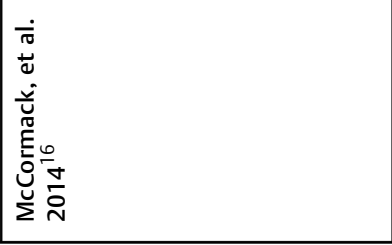 & 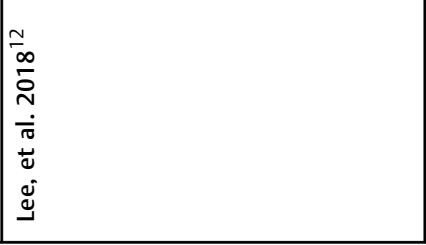 \\
\hline
\end{tabular}


65,058 women were enrolled, showed an inverse association between the amount of caffeine intake and the incidence of tinnitus, with an adjusted hazard ratio of 0.85 (95\% CI: $0.76-0.95$ ) for 450 to $599 \mathrm{mg} /$ day caffeine intake group compared with less than $150 \mathrm{mg} /$ day. Further, higher doses of daily caffeine intake (600 mg or more) were associated with further reduction in the incidence of tinnitus (adjusted hazard ratio $[\mathrm{HR}]=0.79,95 \% \mathrm{CI}$ : 0.68-0.91) compared with the same reference group (less than $150 \mathrm{mg} /$ day), suggesting a dose-dependent effect. ${ }^{15}$ Similarly, the study by McCormack et al. showed, in a cross-sectional population-based survey on individuals aged 40 to 69 years, that both persistent (odds ratio $[\mathrm{OR}]=0.99 ; 95 \%$ confidence interval $[\mathrm{CI}]$ : $0.98-0.99$ ) and transient ( $\mathrm{OR}=0.98 ; 95 \% \mathrm{CI}$ : 0.97-0.99) tinnitus were inversely correlated with the number of cups of coffee consumed daily. ${ }^{16}$ On the other hand, Lee et al., who conducted a cross-sectional study with 13,448 subjects, failed to demonstrate any significant change in the occurrence of tinnitus among daily coffee consumers by reference to rare consumers, both among adults and the elderly. ${ }^{12}$ Conversely, Figueiredo et al. reported that adult individuals with history of bilateral tinnitus and who had a high dose of coffee intake ( $>300 \mathrm{ml} /$ day) were less prone to improvement after 1 -month reduction in caffeine consumption. ${ }^{14}$

Regarding tinnitus severity, no association was observed between caffeine intake and tinnitus-related annoyance among adult (adjusted OR $[\mathrm{aOR}]=0.76 ; 95 \% \mathrm{CI}$ : 0.50, 1.16) and elderly daily consumers ( $\mathrm{aOR}=0.77 ; 95 \% \mathrm{CI}$ : 0.54 , 1.09). ${ }^{12}$ Similarly, report from the UK Biobank cohort study showed no change in the likelihood of bothersome tinnitus $(\mathrm{OR}=0.99 ; 95 \% \mathrm{CI}: 0.97-1.01)$ among participants with persisting tinnitus. $^{16}$

\section{Discussion}

Tinnitus is a common otologic disorder that can affect both male and female individuals at any age. Reduction of caffeine consumption was recommended to relive the symptom of tinnitus in patients with sensorineural hearing loss. ${ }^{14}$ In theory, caffeine is a CNS stimulant, and direct application of caffeine to the inner ear has been shown to reduce the outer hair cell size, and this mechanism may partially account for tinnitus development. ${ }^{17}$ To date, it is unclear whether caffeine can cause or protect against tinnitus. Although tinnitus is subjective and its pathophysiology is unknown, mechanisms are likely to include either hyperactivity of ascending auditory pathways or a reduction in CNS suppression. ${ }^{18}$ Reports included in our analysis did not demonstrate that caffeine consumption increased the risk for tinnitus; rather, they suggested an inverse relationship between caffeine dose intake and tinnitus incidence. ${ }^{15,16}$ The purpose of the present analysis was to systematically review the relationship between caffeine consumption and tinnitus, and the combined conclusions of these reports indicated no increase in the incidence of tinnitus in association with caffeine consumption.
The association of tinnitus with caffeine intake has been investigated by large studies. A large-scale study among caffeine consumers demonstrated that coffee consumption had no direct correlation with tinnitus; but rather some covariates, such as hearing loss, can play an indirect role in the causal pathway. ${ }^{12}$ Although Glicksman, et al. reported an inverse relationship between caffeine intake and tinnitus, they did not control for covariates like hearing difficulty, which compromises the generalization of the conclusion. ${ }^{15}$

Furthermore, caffeine is likely to have an influence on existing tinnitus. This is demonstrated by a randomized controlled trial by Claire et al., which showed no improvement in the symptoms of tinnitus with cessation of caffeine consumption. On the contrary, withdrawal of caffeine could possibly worsen the symptoms of tinnitus. ${ }^{19}$ Figueiredo et al., in their longitudinal cohort that included adults with sensorineural hearing loss with bilateral tinnitus for more than 6 months, demonstrated better improvement in tinnitus index, as a result of caffeine reduction, among younger individuals (aged $<60$ years) with baseline moderate baseline amount of coffee intake (up to $300 \mathrm{ml}$ daily). ${ }^{14}$ However, this study had a relatively low number of subjects, and the aforementioned findings were unadjusted for important confounders that might potentially cause the resultant improvement.

Researchers have continued to study the impact of caffeine on tinnitus-related annoyance. A 2011 study examined coffee consumption in age-related hearing-loss patients with tinnitus. Using a visual analog scale and the Tinnitus Handicap Inventory, no relationship was found between coffee consumption and either degree of discomfort or quality of life related to tinnitus. ${ }^{13}$ Similarly, Lee et al. failed to demonstrate any relationship between coffee consumption and tinnitus annoyance, after adjustment for several covariates including hearing loss. ${ }^{12}$

There was a lack of high quality of evidence in the current literature evaluating the relationship between caffeine intake and tinnitus; thus, it was not possible to establish a causal relationship. Additional estimation errors may have occurred because subjects were asked to estimate (based on memory) their frequency of coffee consumption. In addition to these limitations, a major issue was the small total number of articles relevant to both tinnitus and caffeine.

\section{Final Comments}

To conclude, there is probably a relationship between the incidence of tinnitus and the amount of caffeine intake. Although the data was inconclusive, it appears that caffeine had a protective effect against the development of tinnitus among initially non-afflicted individuals. On the other hand, in patients with tinnitus, a high dose of caffeine intake seems to interfere with the efficacy of caffeine reduction. Further prospective interventional studies are warranted to provide more insight into the relationship between caffeine intake and incidence or severity of tinnitus. 


\section{Authors' Contributions}

Sattam M. Aljuaid: Conception; critical revision for important intellectual content; final approval of the version to be published

Ahmad A. Mirza: Conception; drafting the work; critical revision for important intellectual content; final approval of the version to be published

Lura A. Habib: Drafting the work; final approval of the version to be published

Lujain A. AlHarthi: Drafting the work; final approval of the version to be published

Bashayer M. Alansari: Drafting the work; final approval of the version to be published

Bashaier G. AlQahtani: Drafting the work; final approval of the version to be published

Youssef A. Althobaiti: Drafting the work; final approval of the version to be published

\section{Conflict of Interests}

The authors declare that they have no conflict of interests.

\section{References}

$1 \mathrm{Kim} \mathrm{HJ}$, Lee HJ, An SY, et al. Analysis of the prevalence and associated risk factors of tinnitus in adults. PLoS One 2015;10 (05):e0127578

2 McCormack A, Edmondson-Jones M, Somerset S, Hall D. A systematic review of the reporting of tinnitus prevalence and severity. Hear Res 2016;337:70-79

3 Heller AJ. Classification and epidemiology of tinnitus. Otolaryngol Clin North Am 2003;36(02):239-248

4 Hoare DJ, Kowalkowski VL, Kang S, Hall DA. Systematic review and meta-analyses of randomized controlled trials examining tinnitus management. Laryngoscope 2011;121(07):1555-1564

5 Onishi ET, Coelho CCB, Oiticica J, et al. Tinnitus and sound intolerance: evidence and experience of a Brazilian group. Braz J Otorhinolaryngol 2018;84(02):135-149
6 Pujol R, Puel JL. Excitotoxicity, synaptic repair, and functional recovery in the mammalian cochlea: a review of recent findings. Ann N Y Acad Sci 1999;884:249-254

7 Sepkowitz KA. Energy drinks and caffeine-related adverse effects. JAMA 2013;309(03):243-244

8 John J, Kodama T, Siegel JM. Caffeine promotes glutamate and histamine release in the posterior hypothalamus. Am J Physiol Regul Integr Comp Physiol 2014;307(06):R704-R710

9 Cappelletti S, Piacentino D, Sani G, Aromatario M. Caffeine: cognitive and physical performance enhancer or psychoactive drug? Curr Neuropharmacol 2015;13(01):71-88

10 Ledesma AL, Barreto MA, Bahmad F Jr. Caffeine effect in vestibular system. Int Tinnitus J 2014;19(01):77-81

11 Marmut Z, Belojevic G, Backovic D, Zivojinovic JI, Tomanic M, Hadzic E. Tinnitus among Serbian secondary school students in relation to their behavior and habits. Noise Health 2014;16(69):73-78

12 Lee SY, Jung G, Jang MJ, et al. Association of Coffee Consumption with Hearing and Tinnitus Based on a National Population-Based Survey. Nutrients 2018;10(10):1429

13 Schmidt Rosito LP, Vanni B, Deutsch K, de Brito Belline V. Would Caffeine Consumption Have Some Effect on the Perception of Tinnitus? Otolaryngol Head Neck Surg 2011;145(2_suppl):P233-P233

14 Figueiredo RR, Rates MJ, Azevedo AA, Moreira RK, Penido NdeO. [Effects of the reduction of caffeine consumption on tinnitus perception]. Braz J Otorhinolaryngol 2014;80(05):416-421

15 Glicksman JT, Curhan SG, Curhan GC. A prospective study of caffeine intake and risk of incident tinnitus. Am J Med 2014; 127(08):739-743

16 McCormack A, Edmondson-Jones M, Mellor D, et al. Association of dietary factors with presence and severity of tinnitus in a middleaged UK population. PLoS One 2014;9(12):e114711

17 Trinidade A, Robinson T, Phillips JS. The role of caffeine in otorhinolaryngology: guilty as charged? Eur Arch Otorhinolaryngol 2014;271(08):2097-2102

18 Nehlig A, Daval JL, Debry G. Caffeine and the central nervous system: mechanisms of action, biochemical, metabolic and psychostimulant effects. Brain Res Brain Res Rev 1992;17(02):139-170

19 Claire LS, Stothart G, McKenna L, Rogers PJ. Caffeine abstinence: an ineffective and potentially distressing tinnitus therapy. Int J Audiol 2010;49(01):24-29 